\title{
Improving cytotoxicity against cancer cells by chemo-photodynamic combined modalities using silver-graphene quantum dots nanocomposites
}

This article was published in the following Dove Press journal:

International Journal of Nanomedicine

24 December 2015

Number of times this article has been viewed

\author{
Khaled Habiba ${ }^{1,2}$ \\ Joel Encarnacion-Rosado 2,3 \\ Kenny Garcia-Pabon ${ }^{2,4}$ \\ Juan CVillalobos-Santos ${ }^{2,5}$ \\ Vladimir I Makarov' \\ Javier A Avalos ${ }^{2,6}$ \\ Brad R Weiner $2,7,8$ \\ Gerardo Morell 1,2,7 \\ 'Department of Physics, University of \\ Puerto Rico - Rio Piedras Campus, \\ ${ }^{2}$ Molecular Sciences Research \\ Center, University of Puerto Rico, \\ ${ }^{3}$ Department of Biology, ${ }^{4}$ Faculty \\ of Education, University of Puerto \\ Rico - Rio Piedras Campus, San \\ Juan, ${ }^{5}$ Department of Biology, \\ ${ }^{6}$ Department of Physics, University \\ of Puerto Rico - Bayamon Campus, \\ Bayamon, ${ }^{7}$ Institute for Functional \\ Nanomaterials, University of Puerto \\ Rico, ${ }^{8}$ Department of Chemistry, \\ University of Puerto Rico - Rio \\ Piedras Campus, San Juan, PR, USA
}

Correspondence: Khaled Habiba

Department of Physics, University of

Puerto Rico - Rio Piedras Campus,

309 Natural Sciences (Phase 2) Building,

San Juan, PR 0093I, USA

Tel +I 7877640620

Fax +I 7877644063

Email khaled.habiba@upr.edu

\begin{abstract}
The combination of chemotherapy and photodynamic therapy has emerged as a promising strategy for cancer therapy due to its synergistic effects. In this work, PEGylated silver nanoparticles decorated with graphene quantum dots (Ag-GQDs) were tested as a platform to deliver a chemotherapy drug and a photosensitizer, simultaneously, in chemo-photodynamic therapy against HeLa and DU145 cancer cells in vitro. Ag-GQDs have displayed high efficiency in delivering doxorubicin as a model chemotherapy drug to both cancer cells. The Ag-GQDs exhibited a strong antitumor activity by inducing apoptosis in cancer cells without affecting the viability of normal cells. Moreover, the Ag-GQDs exhibited a cytotoxic effect due to the generation of the reactive singlet oxygen upon $425 \mathrm{~nm}$ irradiation, indicating their applicability in photodynamic therapy. In comparison with chemo or photodynamic treatment alone, the combined treatment of Ag-GQDs conjugated with doxorubicin under irradiation with a $425 \mathrm{~nm}$ lamp significantly increased the death in DU145 and HeLa. This study suggests Ag-GQDs as a multifunctional and efficient therapeutic system for chemo-photodynamic modalities in cancer therapy.
\end{abstract}

Keywords: multifunctional nanoparticles, silver nanoparticles, cancer therapy, drug delivery, photodynamic therapy, nanocomposites

\section{Introduction}

Chemotherapy is one of the most commonly used cancer therapeutic modalities but its limitations include systemic side effects, low efficacy, and drug resistance. ${ }^{1,2}$ Another therapeutic modality, photodynamic therapy (PDT) uses the combined action of a photosensitizer (PS) and specific light sources for the treatment of various cancers. ${ }^{3}$ Upon activation of the PS by a photochemical reaction, reactive oxygen species (ROS) are generated from the molecular oxygen and act to destroy cancer cells. ${ }^{3,4}$ However, clinical applications of PDT have been hindered by limited aqueous solubility and tumor specificity of PSs, and PDT has failed to treat all types of cancer. ${ }^{5,6}$ Other modalities, such as radiotherapy, have displayed severe adverse effects and risks associated with the use of ionizing radiation. ${ }^{7}$ Cancer surgery is not always efficient and suitable for all cases and in particular when the tumor metastasizes and requires further treatment. ${ }^{8}$ Therefore, to increase efficacy and efficiency in treating tumors, integration of multiple treatment strategies is recommended. ${ }^{9}$

In order to apply multiple therapeutic approaches effectively, it is important to choose components with specific intrinsic properties that will not just combine, but that will interact synergistically to produce the desired effect. Recent advances in the synthesis of nanomaterials have led to the development of a number of new drug systems that allow 
multiple therapeutic methods in a single carrier system. ${ }^{1,2,9-11}$ Among them, several multifunctional nanomaterials such as gold nanoparticles, ${ }^{9,12,13}$ semiconductor quantum dots, ${ }^{14,15}$ carbon nanomaterials, ${ }^{10,16-22}$ and magnetic nanoparticles ${ }^{23,24}$ have been explored for theranostic applications.

Graphene and graphene-based materials, ie, graphene oxide (GO) and graphene quantum dots (GQDs), have attracted interest in the biomedical field and have shown great potential for making a significant impact in cancer therapy and diagnosis. ${ }^{10,16-22,25-30}$ GQDs demonstrated excellent electrochemical and optical properties as well as the capability to bind with a variety of aromatic biomolecules through a $\pi-\pi$ stacking interaction and/or electrostatic interaction, which make them ideal materials for loading chemotherapeutic drugs. ${ }^{18,19,21,27,30}$ The use of GQDs to deliver doxorubicin (DOX), a chemotherapy drug, to cancer cells was reported, and the release of the anticancer drug was $\mathrm{pH}$ responsive. ${ }^{19,30}$ Wang et al showed that GQDs delivered DOX to the nucleus of the cancer cells and enhanced the cytotoxicity of DOX dramatically. ${ }^{18}$ They showed that GQDs do not release their cargo through a $\mathrm{pH}$-triggering mechanism and postulated that DOX binds to the nuclear DNA after the diffusion of GQDs to the nucleus of the cell. Moreover, the use of GQDs in PDT was reported, with demonstrated cytotoxicity upon irradiation with visible light. ${ }^{16,17}$

Silver nanoparticles (Ag-NPs) are another class of nanomaterials that have been used in the biomedical field, ie, as antibacterial agents and anticancer agents. ${ }^{31-33}$ The Food and Drug Administration (FDA) approved Ag-NPs-based bandage for treating burn wounds, known under the trade name Acticoat, is already in commercial use. ${ }^{32}$ Franco-Molina et al reported that colloidal silver has antitumor activity against breast cancer cells. ${ }^{31}$ Sanpui et al showed that a small amount of Ag-NPs delivered by chitosan nanoparticles can induce apoptosis in cancer cells, and display anti-proliferative activity. ${ }^{33}$ However, silver nanoparticles have shown genotoxicity, and Asharani et al reported that their use should be administered at low doses to avoid toxicity in healthy cells. ${ }^{34}$

Synthesis of nanocomposites has been established to enhance the intrinsic properties of nanoparticles, and to overcome the adverse effects associated with the core material of the nanocomposites, through the decoration or encapsulation of its surface. Synthesis of carbon-metallic nanocomposites and their use in the biomedical field has been reported previously. ${ }^{35-38}$ Such synthetic strategies include PEGylation, a process that involves the passivation of the surface of nanoparticles by polyethylene glycol (PEG), to enhance their dispersion and solubility in aqueous solutions. ${ }^{39,40}$ It has been

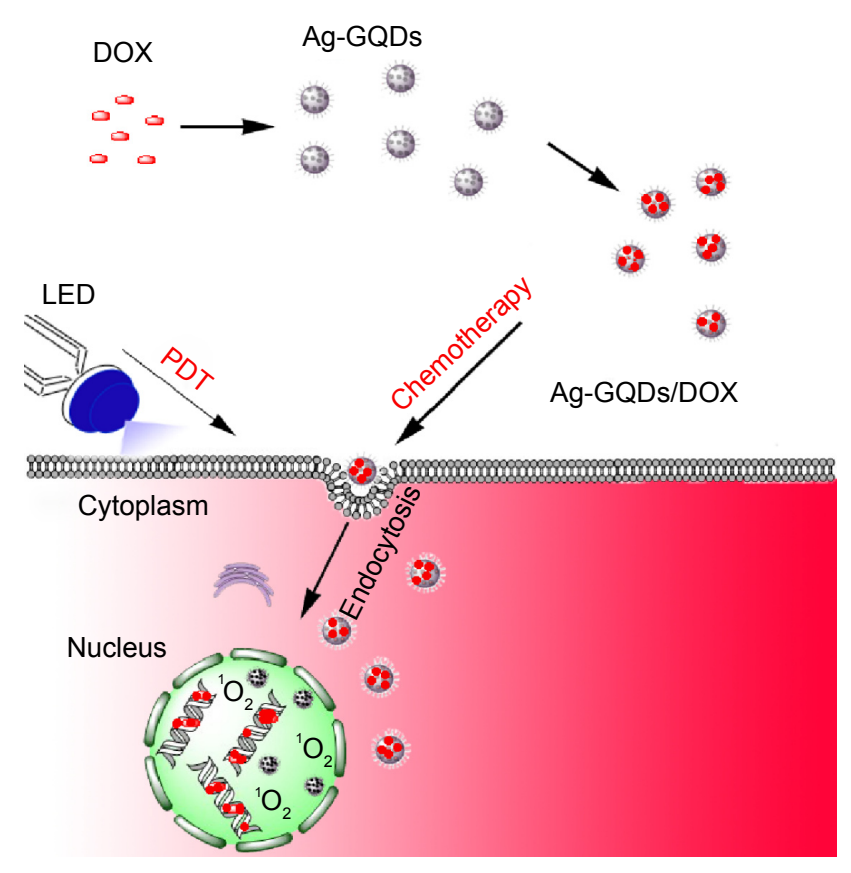

Figure I Schematic illustrating the preparation of Ag-GQDs/DOX nanoconjugates and their use in chemo-photodynamic combined modalities in improving cancer therapy.

Abbreviations: Ag-GQDs, silver nanoparticles decorated with graphene quantum dots; Ag-GQDs/DOX, silver nanoparticles decorated with graphene quantum dots conjugated with doxorubicin; DOX, bare doxorubicin; LED, light emitting diode; PDT, photodynamic therapy.

reported that the interaction of the reticuloendothelial system, ie, macrophages and monocytes with PEGylated nanoparticles decreases, and their circulation time in blood increases. ${ }^{39}$

In this work, we report the design of anticancer nanoconjugates by decorating Ag-NPs with GQDs and coupling them with DOX to form silver-GQDs conjugated with DOX (Ag-GQDs/DOX; Figure 1). The nanoconjugates were used to treat two different cancer cell lines and comprised a combination of chemo-photodynamic modalities in treatment. By taking the advantage of each component of the nanoconjugates, it is anticipated that the resulting silver nanoparticles decorated with GQDs (Ag-GQDs)/DOX can significantly enhance the therapeutic efficiency by using multiple mechanisms to kill cancer cells.

\section{Methods and materials Synthesis of Bare GQDs and Ag-GQDs}

For the synthesis of bare GQDs, we used our bottom-up synthesis approach as described previously. ${ }^{41,42}$ To functionalize the synthesized bare GQDs with PEG, we modified the irradiated mixture as follows. A $0.25 \mathrm{wt} \%$ mixture of nickel oxide powder (Alfa Aesar, Ward Hill, MA, USA) and $1.25 \mathrm{wt} \%$ of PEG bis (3-aminopropyl) terminated (Sigma-Aldrich St Louis, MO, USA) in $98.5 \mathrm{wt} \%$ benzene (Sigma-Aldrich) was irradiated for 
45 minutes with a 1,064 nm pulsed Nd:YAG laser ([Continuum Surelite II, San Jose, CA, USA] KDP doubling crystal, $10 \mathrm{~Hz}$, $10 \mathrm{~ns}$ pulse width). The synthesized GQDs were separated from benzene and the precipitated nickel oxide by centrifugation at $9,030 \times g$, following the dissolution in nano-pure water. Finally, the GQDs solution was purified by using dialysis bags.

For the synthesis of Ag-GQDs, a mixture of $0.25 \mathrm{wt} \%$ of silver powder (Sigma-Aldrich) and $1.25 \mathrm{wt} \%$ of PEG bis(3-aminopropyl) terminated (Sigma-Aldrich) in $98.5 \mathrm{wt} \%$ benzene (Sigma-Aldrich) was prepared and irradiated by pulsed laser, similar to the synthesis of bare GQDs. The synthesized Ag-GQDs nanoparticles were separated from benzene and dissolved in nano-pure water, and purified by using dialysis bags.

\section{Characterization techniques}

High-resolution transmission electron microscopy (HR-TEM) images were recorded using an electronic microscope (JEOL JEM-2200FS, JEOL Ltd., Tokyo, Japan), operated at $200 \mathrm{kV}$ in scanning transmission electron microscopy mode. The UVvisible spectra were recorded on a spectrophotometer (Shimadzu 2550, Kyoto, Japan). X-ray diffraction (XRD) patterns were obtained with an instrument (Rigaku Smart-lab, Rigaku Corporation, Tokyo, Japan), equipped with a $\mathrm{Cu} \mathrm{K}_{\alpha}$ radiation source, at an accelerating potential of $40 \mathrm{kV}$ and a tube current of $44 \mathrm{~mA}$. The XRD samples were prepared by placing a certain amount of Ag-GQDs powder on a silicon substrate. The hydrodynamic size and zeta potential of the Ag-GQDs nanocomposites before and after conjugation with DOX were determined by dynamic light scattering (DLS) using a Malvern Zetasizer Nanoseries (Malvern Instruments, Malvern, UK). The samples were dispersed in phosphate-buffered saline (PBS) solution at $\mathrm{pH}=5$ or 7.4 and sonicated until a good suspension was obtained. For zeta potential experiments in different media, Ag-GQDs were suspended in PBS or Eagle's minimum essential medium (EMEM) supplied with $10 \%$ fetal bovine serum (FBS) at a $\mathrm{pH}=7.4$. For photoluminescence $(\mathrm{PL})$ emission experiments of the Ag-GQD solutions were recorded using a Shimadzu RF-5301 spectrofluorometer equipped with a Xenon lamp as an excitation source.

\section{Preparation of Ag-GQDs/DOX nanoconjugates}

Stock solutions of Ag-GQDs/DOX or GQDs/DOX were prepared by mixing $1 \mu \mathrm{M}$ of DOX with different concentrations of Ag-GQDs or GQDs dissolved in PBS (ie, 25, 50, 75, 100,150 , and $200 \mu \mathrm{g} / \mathrm{mL}$ ) and incubating for 40 minutes in the dark at room temperature. ${ }^{18}$

\section{Characterization of Ag-GQDs/DOX nanoconjugates}

$\mathrm{UV}-\mathrm{V}$ is absorption and PL emission of the mixtures were recorded. The binding constant of DOX to Ag-GQDs was calculated by monitoring the quenching in the fluorescence of DOX according to a previously published method. ${ }^{43}$ The fluorescence spectra of DOX were recorded at $\lambda_{\mathrm{ex}}=500 \mathrm{~nm}$ and $\lambda_{\text {em }}$ from 520 to $700 \mathrm{~nm}$ while increasing the concentration of Ag-GQDs.

\section{Cell culture}

DU145 and HeLa cells were cultured in EMEM (ATCC, Manassas, VA, USA) supplemented with 10\% FBS (ATCC), $100 \mathrm{U} / \mathrm{mL}$ penicillin, $100 \mu \mathrm{g} / \mathrm{mL}$ streptomycin, and $250 \mathrm{ng} / \mathrm{mL}$ amphotericin B (Cellgro, Manassas, VA, USA) at $37^{\circ} \mathrm{C}$ with $5 \% \mathrm{CO}_{2}$. No ethics statement was required from the institutional review board for the use of these cell lines.

\section{Evaluation of the chemotherapy in HeLa and DUI 45 with the nanoconjugates using Ag-GQDs/DOX}

For the treatment of cells with Ag-GQDs/DOX, we prepared fresh solutions of Ag-GQDs/DOX by mixing $1 \mu \mathrm{M}$ of DOX with different concentrations of Ag-GQDs dissolved in EMEM serum-free media (ie, 75, 100, and $200 \mu \mathrm{g} / \mathrm{mL}$ ) and left them to incubate for 40 minutes in the dark at room temperature. ${ }^{18}$ Similarly, we prepared GQDs/DOX nanoconjugates as a comparative control at the same concentration of DOX and GQDs. The viability of DU145 and HeLa cells in the presence of DOX, bare GQDs, Ag-GQDs, GQDs/DOX, or Ag-GQDs/ DOX was evaluated using the (3-(4,5-dimethylthiazol-2-yl)5-(3-carboxymethoxyphenyl)-2-(4-sulfophenyl)-2Htetrazolium)-based (MTS) CellTiter $96{ }^{\circledR}$ AQueous Solution Cell Proliferation Assay (Promega, Fitchburg, WI, USA). Cells were plated in a 96-well microplate at a density of $10^{4}$ cells and left to incubate overnight at $37^{\circ} \mathrm{C}$. The media cells were then treated with Ag-GQDs, GQDs, DOX, GQDs/ DOX, or Ag-GQDs/DOX, and the plates were incubated for an additional 24 hours. Fresh culture medium was used as a negative control. After treatment, the medium was discarded and replaced by $100 \mu \mathrm{L}$ of fresh cell medium supplemented with $20 \mu \mathrm{L}$ of MTS reagent. The cells were then incubated for 30 minutes at $37^{\circ} \mathrm{C}$ and centrifuged at $380 \times g$ for 5 minutes. Subsequently, the cell medium containing the MTS reagent was transferred to a new microplate and the absorbance at $490 \mathrm{~nm}$ was measured with a UV-Vis microplate spectrometer (Biotek Synergy H4 Hybrid, BioTek Instruments, Inc., Winooski, VT, USA). 


\section{Evaluation of the cell viability of Ag-GQDs in normal cells}

Vero cells (ATCC, Manassas, VA, USA; obtained from the Center for Disease Control and Prevention-Dengue Branch) were maintained in M199 medium (Mediatech, Manassas, VA, USA) containing 5\% heat-inactivated FBS, $1 \%$ sodium bicarbonate, $1 \%$ Hepes buffer, $1 \%$ glutamine, and $1 \%$ penicillin-streptomycin at $5 \% \mathrm{CO}_{2}$ and $37^{\circ} \mathrm{C}$. The cell viability effects of Ag-GQDs nanoparticles were assessed on Vero by using the MTS CellTiter $96^{\circledR}$ AQueous Solution Cell. In 96-well plates (Falcon), $10^{4}$ cells were seeded and grown overnight. Cells were treated with bare Ag-GQDs at the concentrations (25, 50, 100, 150, 400, and $600 \mu \mathrm{g} / \mathrm{mL}$ ). After 24 hours of incubation of the cells, the media were then discarded, and $100 \mu \mathrm{L}$ of fresh cell medium with $20 \mu \mathrm{L}$ of MTS reagent was added. The cells were incubated for 120 minutes at $37^{\circ} \mathrm{C}$ and centrifuged at $380 \times g$ for 5 minutes. Subsequently, the cell medium containing the MTS reagent was transferred to a new microplate and the absorbance at $490 \mathrm{~nm}$ was measured with a UV-Vis microplate spectrometer.

\section{Cell apoptosis assays}

Caspase-3/7 activities were measured using the Apo-ONE Homogeneous Caspase-3/7 Assay kit (Promega) according to the manufacturer's protocol. HeLa and DU145 cells were plated in triplicate in 96-well cell culture plates (COSTAR, CORNING) at a density of $10^{4}$ cells and incubated overnight at $37^{\circ} \mathrm{C}$. Subsequently, cells were treated with Ag-GQDs (100 $\mu \mathrm{g} / \mathrm{mL})$, GQDs $(100 \mu \mathrm{g} / \mathrm{mL})$, DOX $(1 \mu \mathrm{M})$, Ag-GQDs/ DOX $(1 \mu \mathrm{M}$ of DOX with $100 \mu \mathrm{g} / \mathrm{mL})$ or GQDs/DOX (1 $\mu \mathrm{M}$ of DOX with $100 \mu \mathrm{g} / \mathrm{mL}$ ), and fresh cell medium was used as a negative control. After 24 hours of treatment, the cells were lysed with buffer containing caspase substrate Z-DEVDR100, and incubated at room temperature until they were analyzed. As a control, non-treated cells were lysed with buffer containing caspase substrate Z-DEVD-R100, and incubated at room temperature until analysis. Assays were measured by detection with a fluorescence microplate reader, and the fluorescence was measured at an excitation/emission wavelength of $485 / 535 \mathrm{~nm}$. The results are presented as the mean \pm standard deviation (SD) of the triplicates.

\section{Fluorescence microscopy}

DU145 cells were plated at a density of $4 \times 10^{4}$ cells per well in six-well plates to culture sequentially. After 24 hours, the cells were treated with the medium without serum but containing $1 \mu \mathrm{M}$ of DOX with $100 \mu \mathrm{g} / \mathrm{mL}$ of Ag-GQDs followed by incubation for 18 hours, and then washing with PBS buffer two times. For a negative control, non-treated cells were incubated in the fresh medium without serum and DOX alone $(1 \mu \mathrm{M})$ and bare Ag-GQDs ( $100 \mu \mathrm{g} / \mathrm{mL})$ were used as a comparative control. After washing with PBS, fresh serum-free medium was added to each well and cells' nuclei were stained using Hoechst 33342 (NucBlue, Thermo Fisher Scientific, Waltham, MA, USA) for 20 minutes. The cells were washed two times with PBS buffer to remove the residual staining dye. The excitation/emission measurements for Hoechst 33342 and DOX was 380/460 and 500/560 nm, respectively. The plates were imaged using a fluorescence microscope (Olympus BX51WI, Tokyo, Japan) equipped with a CMOS camera, Hamamatsu ORCA Flash 4 (Hamamatsu Photonics K.K., Hamamatsu, Japan), the images were recorded on a magnification of $40 \times$, and the excitation wavelengths used were 380 and $500 \mathrm{~nm}$.

\section{Qualitative analysis of singlet oxygen and evaluation of PDT in vitro}

To evaluate the singlet oxygen generation by visible light irradiation of GQDs and Ag-GQDs, the singlet oxygen sensor green (SOSG, Thermo Fisher Scientific) at a concentration of $6 \mu \mathrm{M}$ was mixed with GQDs or Ag-GQDs in PBS prepared at a concentration of $300 \mu \mathrm{g} / \mathrm{mL}$ in a final volume of $150 \mu \mathrm{L}$. Singlet oxygen generation was induced by irradiation with a light intensity of $3 \mathrm{~mW} / \mathrm{cm}^{2}$ using a $425 \pm 10 \mathrm{~nm}$ light emitting diode (LED; Shenzhen Dongzhiyao Lighting Technology Co., Ltd., Guang Dong, People's Republic of China). After irradiation, SOSG fluorescence was measured at an excitation and emission of 488 and $525 \mathrm{~nm}$, respectively, using a microplate reader (Synergy H4 Hybrid, BioTek Instruments, Inc.,). PBS with SOSG was irradiated and served as a negative control. All samples were irradiated together and the readings were taken at the same time interval every 5 minutes.

To compare the dark toxicity and phototoxicity of the GQDs and Ag-GQDs, the GQD or Ag-GQDs stock solutions were diluted with fresh medium to various concentrations (75, 100, and $200 \mu \mathrm{g} / \mathrm{mL}$ ). In 96-well plates (Falcon), $10^{4}$ cells (DU145 or HeLa) were seeded and grown overnight. The cell medium was then exchanged for different concentrations of Ag-GQDs or GQDs medium solution. The cells were then incubated with these solutions at $37^{\circ} \mathrm{C}$ in $5 \% \mathrm{CO}_{2}$ for 18 hours, and the cultures were then irradiated at $3 \mathrm{~mW} / \mathrm{cm}^{2}$ using a $425 \pm 10 \mathrm{~nm}$ LED lamp for 15 minutes before removing the GQDs or Ag-GQDs solution and adding fresh medium. Subsequently, the plates were incubated at $37^{\circ} \mathrm{C}$ in $5 \% \mathrm{CO}_{2}$ for 6 hours. The cell medium solutions were exchanged for $100 \mu \mathrm{L}$ of fresh medium, followed by 
the addition of $20 \mu \mathrm{L}$ of the MTS solution to each well. The culture plates were then incubated at $37^{\circ} \mathrm{C}$ in $5 \% \mathrm{CO}_{2}$ for 40 minutes. The cell medium containing the MTS reagent was transferred to a new microplate and the absorbance at $490 \mathrm{~nm}$ was measured with a UV-Vis microplate spectrometer.

\section{Evaluation of the chemo-photodynamic combined therapy in vitro}

To evaluate the enhancement in the therapeutic efficiency of treatment of DU145 and HeLa cells with the nanoconjugates, we assessed the concentration-dependent cell viability assay for the cells treated with Ag-GQDs under irradiation, AgGQDs/DOX in the dark and Ag-GQDs/DOX under irradiation. In 96-well plates (Falcon), $10^{4}$ cells (DU145 or HeLa) were seeded and grown overnight. The cells were then treated with the nanoparticles as described earlier. Fresh cell medium culture was used as a negative control, and for comparison we used cells treated with DOX at $1 \mu \mathrm{M}$. The cells were then incubated with these solutions at $37^{\circ} \mathrm{C}$ in $5 \% \mathrm{CO}_{2}$ for 18 hours, and the cultures were then irradiated at $3 \mathrm{~mW} / \mathrm{cm}^{2}$ using a $425 \pm 10 \mathrm{~nm}$ light-emitting lamp for 15 minutes (for the irradiated test group) before removing the Ag-GQDs or Ag-GQDs/DOX solution and adding fresh medium. For the test group in the dark, we protected the wells from the light while irradiating the plate. Subsequently, the plates were incubated at $37^{\circ} \mathrm{C}$ in $5 \% \mathrm{CO}_{2}$ for 6 hours. The cell medium solutions were exchanged for $100 \mu \mathrm{L}$ of fresh medium, followed by the addition of $20 \mu \mathrm{L}$ of the MTS solution to each well. The culture plates were then incubated at $37^{\circ} \mathrm{C}$ in $5 \%$ $\mathrm{CO}_{2}$ for 40 minutes. The cell culture medium containing the MTS reagent was transferred to a new microplate, and the absorbance at $490 \mathrm{~nm}$ was measured with a UV-Vis microplate spectrometer.

\section{Statistical analysis}

Statistical analyses were conducted between treated and nontreated groups using analysis of variance, and Student's $t$-test statistical analysis was used to compare two groups together (eg, Ag-GQDs and Ag-GQDs/DOX or GQDs and GQDs/ DOX), and $P$-values obtained are as follows: $\left.{ }^{*} P<0.05\right)$, $* * P<0.01$, and $* * * P<0.001$ are considered as significant.

\section{Results and discussion Synthesis and characterization of Ag-GQDs}

We employed the pulsed laser synthesis technique to synthesize the PEGylated Ag-GQDs in a single step. ${ }^{42}$ Briefly, a mixture of silver nanoparticles, PEG, and benzene was prepared and then irradiated by a pulsed laser for 45 minutes. Ag-NPs were used as a catalyst for the reaction and as a source for silver nanocomposites. The resulting nanoparticles were separated from benzene, purified, and dissolved in nano-pure water.

The HR-TEM images (Figure 2A) show particles less than $10 \mathrm{~nm}$ in diameter. Figure 2B depicts prepared Ag-GQDs composite at higher magnification. It is observed that GQDs (point out by yellow font) are attached on the surface of Ag nanoparticles, where the d-spacing planes of Ag and GQDs (marked in white font) can be distinguished. The d-spacing of $0.145 \mathrm{~nm}$ corresponds to the (220) plane of silver, ${ }^{44}$ and the d-spacing of $0.214 \mathrm{~nm}$ corresponds to the (110) plane of GQDs. ${ }^{41,45}$

We used the XRD technique to identify the presence of silver and GQDs in the nanocomposites. The XRD pattern (Figure 3A) of the Ag-GQDs powder shows the presence of nine peaks. The peaks at $38.1^{\circ}, 44.3^{\circ}, 64.5^{\circ}$, and $77.5^{\circ}$ are assigned to the crystallographic planes (111), (200), (220), and (311) of the face-centered cubic Ag nanoparticles, respectively, consistent with the Joint Committee on Powder Diffraction Standards card No. 04-0783. ${ }^{44}$ The peaks shown at $18.97^{\circ}, 23.3^{\circ}$, and $35.7^{\circ}$ are attributed to $\mathrm{PEG},{ }^{46}$ which is conjugated on the surface of Ag-GQDs, and match with the PEG peaks reported by El Moussaoui et al. The observed peaks at $26.6^{\circ}$ and $54.79^{\circ}$ correspond to the d-spacing of the planes (002) and (004) of graphite, respectively, according to the JCPDS card No. $9008569 .{ }^{47}$

The UV-Vis spectrum (Figure 3B) displays an absorption at $\sim 420 \mathrm{~nm}$ corresponding to the localized surface plasmon of silver, ${ }^{48}$ and the absorption at $\sim 270 \mathrm{~nm}$ is attributed to $\pi-\pi^{*}$ transition of aromatic $\mathrm{sp}^{2}$ domains that is commonly observed in graphene and graphite nanostructures. ${ }^{41}$ The $\mathrm{XRD}$ and UV-Vis data are in good agreement with the HRTEM data obtained earlier.
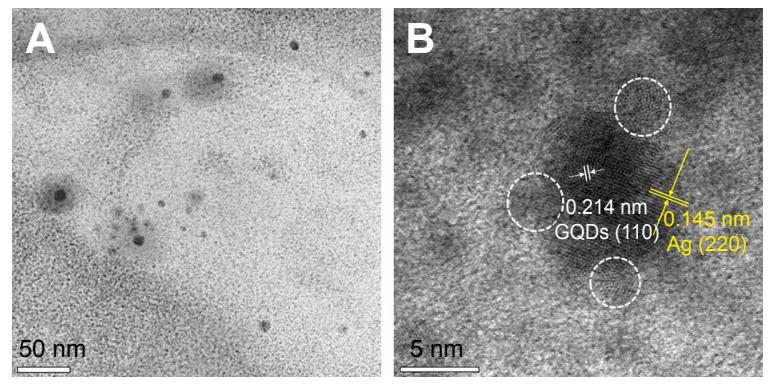

Figure 2 HR-TEM images of the Ag-GQDs.

Notes: (A) Low magnification image shows particles with a diameter less than $10 \mathrm{~nm}$; (B) high magnification image showing a silver nanoparticle decorated with GQDs (pointed out in white circles).

Abbreviations: Ag-GQDs, silver nanoparticles decorated with graphene quantum dots; GQDs, graphene quantum dots; HR-TEM, high-resolution transmission electron microscope. 

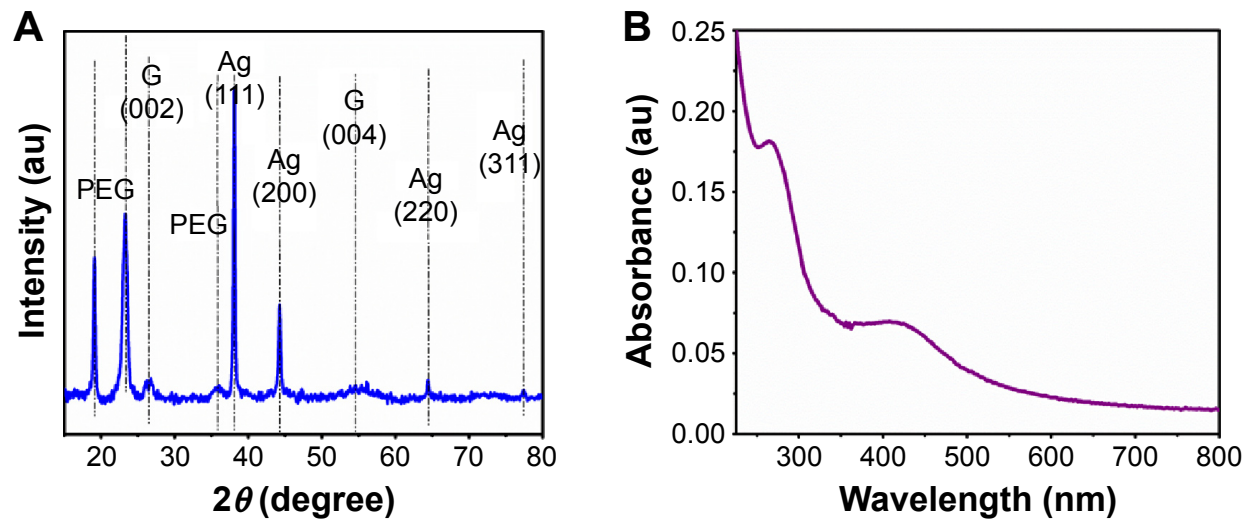

Figure 3 Spectroscopic characterization of Ag-GQDs nanocomposites.

Notes: (A) XRD pattern of Ag-GQDs shows the characteristic peaks of Ag, GQDs, and PEG. (B) UV-Vis spectrum of Ag-GQDs.

Abbreviations: Ag, Silver; au, arbitrary unit; Ag-GQDs, silver nanoparticles decorated with graphene quantum dots; GQDs, graphene quantum dots; PEG, polyethylene glycol; XRD, X-rays diffraction spectroscopy.

\section{Formation of DOX and Ag-GQDs nanoconjugates (Ag-GQDs/DOX)}

The chemotherapy drug DOX was used to evaluate the feasibility of using the PEGylated Ag-GQDs as a drug carrier. DOX was loaded by mixing it with Ag-GQDs in serum-free medium and leaving it to incubate for 40 minutes in the dark. ${ }^{18}$ To verify the loading of the DOX onto the Ag-GQDs nanocomposites, we employed UV-Vis and PL emission spectroscopic techniques to monitor the electronic interactions between them. The UV-Vis spectra of the bare DOX, bare Ag-GQDs, and Ag-GQDs/DOX are shown in Figure 4A. The $\mathrm{UV}-\mathrm{Vis}$ spectrum of the bare DOX shows the presence of the featured peak of DOX at $\sim 500 \mathrm{~nm}$, which shifts to $\sim 512 \mathrm{~nm}$ upon the conjugation onto the surface of Ag-GQDs. Wang et al reported that the shift in the absorption peak may be due to the $\pi-\pi$ stacking and hydrophobic interactions between DOX and the abundant aromatic rings in GQDs. ${ }^{19}$

Moreover, we monitored the PL emission of DOX upon conjugation with Ag-GQDs to determine the binding constant of the DOX to Ag-GQDs using the previously described procedures (Figure 4B). ${ }^{43}$ The quenching of the fluorescence of DOX upon increasing the concentrations of Ag-GQDs, most likely due to the binding of more molecules of DOX onto the nanoparticles, was observed. Quenching of PL emission of DOX upon conjugation of GQDs located on the surface of Ag-GQDs with DOX due to the $\pi-\pi$ stacking has been reported, ${ }^{18}$ consistent with the UV-Vis data obtained here. The binding constant of the DOX to Ag-GQDs is $0.261 \mu \mathrm{M}^{-1}$.
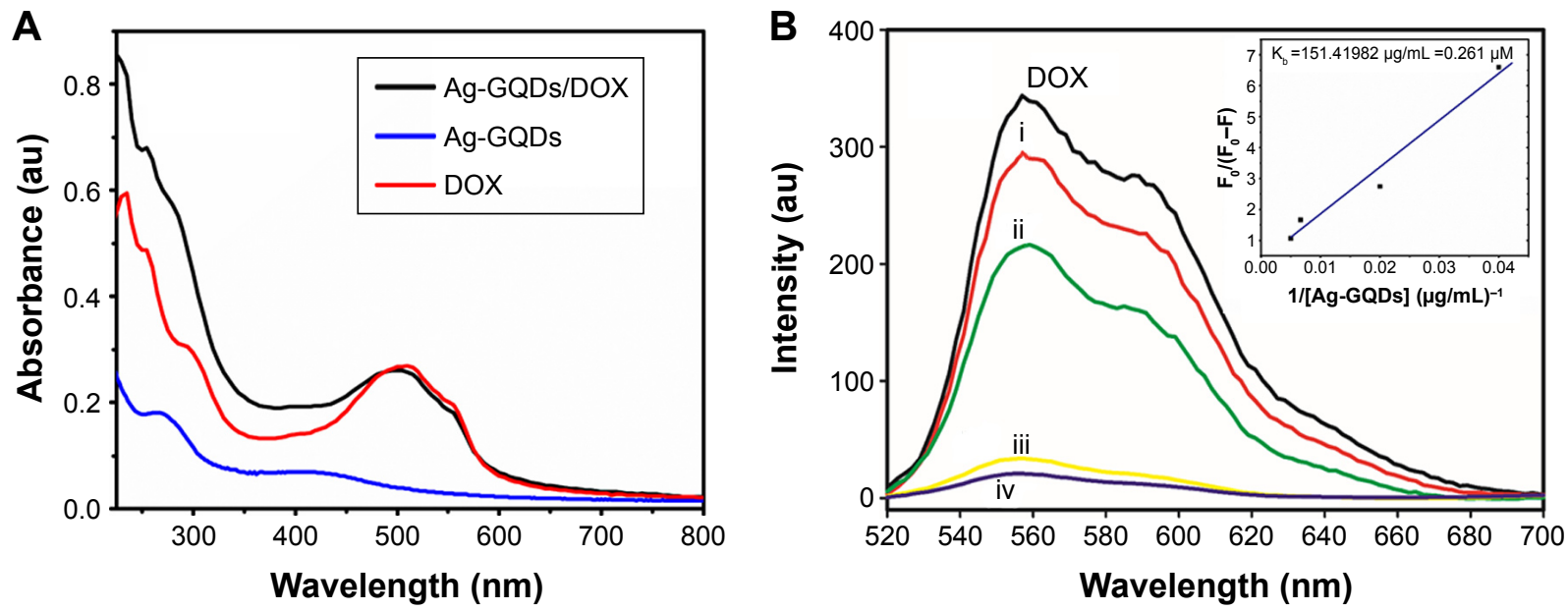

Figure 4 Characterization of Ag-GQDs/DOX.

Notes: (A) UV-Vis absorption spectra of Ag-GQDs, DOX, and Ag-GQDs/DOX; (B) fluorescence of DOX (I $\mu$ M) is quenched by the increasing amount of Ag-GQDs from 25 to $200 \mu \mathrm{g} / \mathrm{mL}$ (i. 25 , ii. 50 , iii. I 50, and iv. $200 \mu \mathrm{g} / \mathrm{mL}$ ) in PBS buffer (pH 7.4). Excitation wavelength was $500 \mathrm{~nm}$.

Abbreviations: Ag-GQDs/DOX, silver nanoparticles decorated with graphene quantum dots conjugated with doxorubicin; Ag-GQDs, silver nanoparticles decorated with graphene quantum dots; au, arbitrary unit; DOX, bare doxorubicin; PBS, phosphate-buffered saline. 
The physicochemical properties of nanoparticles, such as hydrodynamic particle size and surface charge, play a key role in their cellular uptake. Thus, we investigated the physicochemical properties of the Ag-GQDs/DOX using DLS and zeta potential techniques. Figure $5 \mathrm{~A}$ and $\mathrm{B}$ shows the change in the mean diameter of Ag-GQDs from 33 to $64 \mathrm{~nm}$ upon their conjugation with DOX. Nanoparticles greater than 6-8 $\mathrm{nm}$ in effective hydrodynamic diameter are subjected to fast renal clearance while those $\sim 50-60 \mathrm{~nm}$ in hydrodynamic diameter often exhibit optimal cellular uptake and intra-tumor accumulation. ${ }^{49}$ The polydispersity index obtained for AgGQDs and Ag-GQDs/Dox is 0.095 and 0.099, respectively, indicating that their solutions are monodispersed. Therefore, it is anticipated that the Ag-GQDs/DOX nanoconjugates in this study may be suitable for cancer therapy and drug delivery.

We tested the zeta potential of the Ag-GQDs before and after conjugation with DOX at pH of 7.4 and 5 (Figure 5C). It can be observed that the zeta potential of Ag-GQDs decreases upon the conjugation with DOX and confirms the interaction between them. The magnitude of the zeta potential of Ag-GQDs/DOX nanoconjugates decreases from approximately -8.5 to $-2.5 \mathrm{mV}$. Li et al reported that polyplexes nano-vehicles prolong their circulation under a neutral environment (ie, $\mathrm{pH}=7.4$ ), such as in the bloodstream, until reaching an acidic environment such as a tumor tissue (ie, $\mathrm{pH}$ less than 6.8), where they accumulate inside and the cargo is most likely delivered to the tumor cells. ${ }^{50}$ Moreover, we performed measurements for Ag-GQDs/DOX conjugates at neutral $\mathrm{pH}$ in both PBS and EMEM supplied with $10 \%$ FBS, finding that the zeta potential of the nanoconjugates did not change (ie, $\sim 8.5 \mathrm{mV}$ ). This result indicates that the PEGylation of Ag-GQDs protected their surface and efficiently reduced their surface charge, while decreasing the adsorption of proteins in FBS on their surface. ${ }^{51}$ Therefore, Ag-GQDs are anticipated to have prolonged circulation in the bloodstream and reduced interactions with non-specific targets such as macrophages..$^{51}$

\section{Testing Ag-GQDs as nanocarriers for chemotherapy drug in vitro in cancer cells} The in vitro cytotoxicity of the Ag-GQDs/DOX nanoconjugates was tested in human cervical cancer cells HeLa and
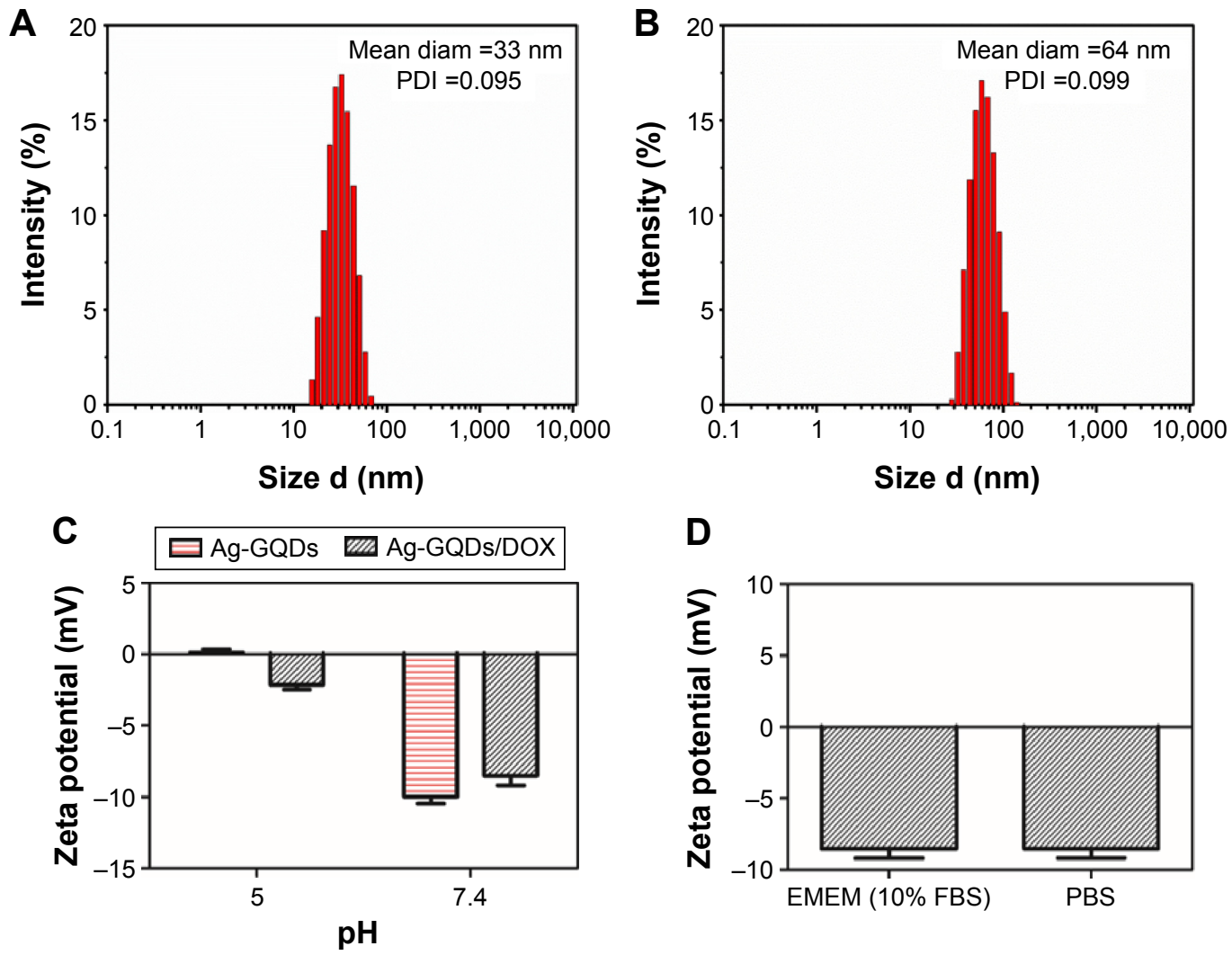

D

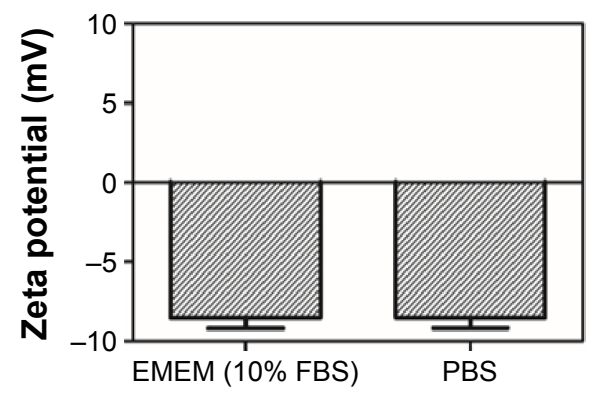

Figure 5 Physicochemical characterization of Ag-GQDs before and after conjugation with DOX.

Notes: (A) Hydrodynamic diameter of Ag-GQDs; (B) hydrodynamic diameter of Ag-GQDs/DOX; (C) zeta potential of Ag-GQDs and Ag-GQDs/DOX at pH =7.4 and 5; (D) zeta potential of Ag-GQDs in different media (ie, EMEM containing FBS and PBS).

Abbreviations: Ag-GQDs/DOX, silver nanoparticles decorated with graphene quantum dots conjugated with doxorubicin; Ag-GQDs, silver nanoparticles decorated with graphene quantum dots; DOX, bare doxorubicin; EMEM, Eagle's minimum essential medium; FBS, fetal bovine serum; PBS, phosphate-buffered saline; PDI, polydispersity index; diam, diameter. 

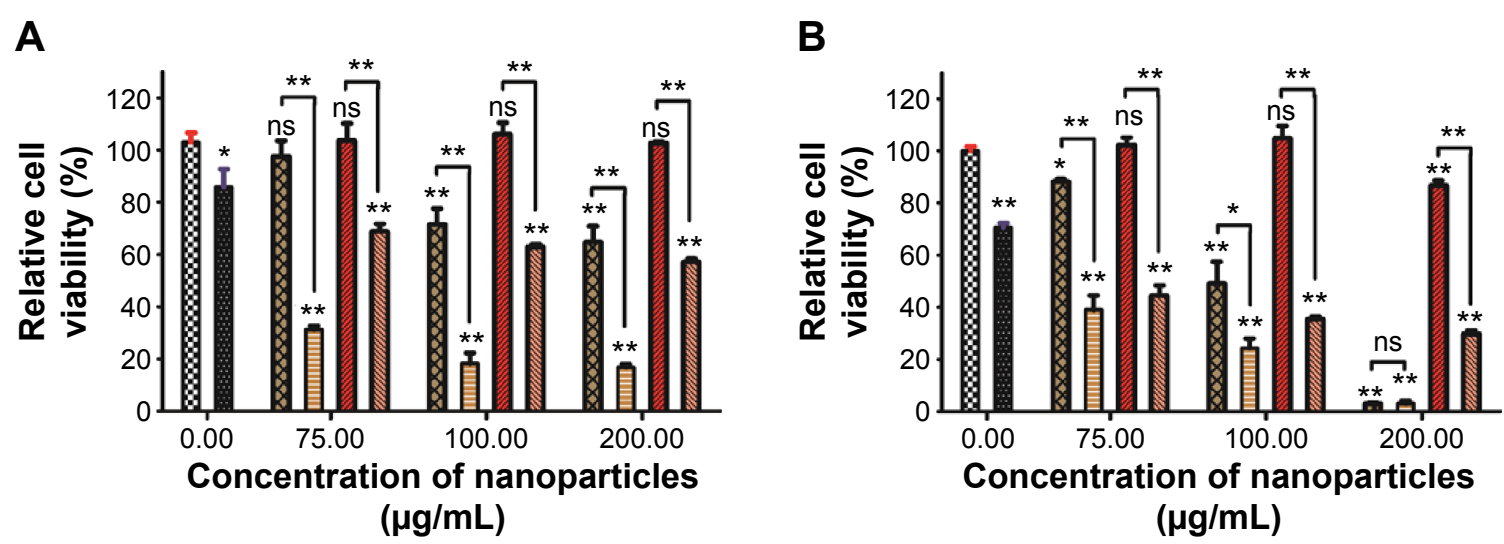

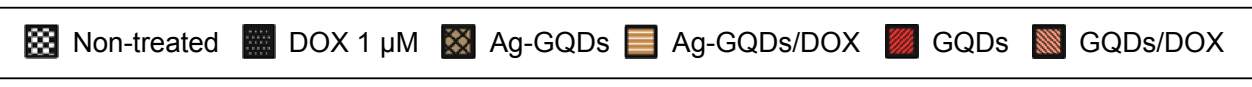

Figure 6 Relative cell viability of cells after treatment for 24 hours with Ag-GQDs, Ag-GQDs/DOX, GQDs, GQDs/DOX, and free DOX (A) HeLa cells. (B) DUI45 cells. Notes: $P$-values are shown as follows: ns, non-significant, $* P<0.01$, and $* * P<0.001$ are considered as significant.

Abbreviations: Ag-GQDs/DOX, silver nanoparticles decorated with graphene quantum dots conjugated with doxorubicin; DOX, bare doxorubicin; Ag-GQDs, silver nanoparticles decorated with graphene quantum dots; GQDs, graphene quantum dots; GQDs/DOX, graphene quantum dots conjugated with doxorubicin.

prostate cancer cells DU145 using the MTS cell proliferation assay after 24 hours of treatment. To evaluate the efficiency of Ag-GQDs as chemotherapeutic agents and their high potential in drug delivery, we tested them in comparison to GQDs conjugated with DOX (GQDs/DOX), bare GQDs (control for GQDs/DOX), and DOX alone. The cell viability results (Figure 6) show that the $1 \mu \mathrm{M}$ of free DOX decreased the cell proliferation by only $\sim 10 \%$ and $\sim 20 \%$ in HeLa and DU145 cancer cells, respectively. The bare GQDs for all tested concentrations did not show any cytotoxic effect against cancer cells. For bare Ag-GQDs, they showed a dose-dependent cytotoxicity against both cancer cells, which may be due to the anticancer activity associated with silver. Sanpui et al reported that silver nanoparticles decrease the cell proliferation and induce apoptosis in cancer cells. ${ }^{33}$ Looking into the feasibility of GQDs and Ag-GQDs as drug vehicles, it can be observed that both of them efficiently delivered their cargo of DOX to the cancer cells (Figure 6). However, the treatment efficacy with Ag-GQDs is much higher as compared to the treatment with GQDs at the same concentration, and the delivery efficiency was observed higher in HeLa than in DU145. However, DU145 were more sensitive to Ag-GQDs than HeLa cells and the $200 \mu \mathrm{g} / \mathrm{mL}$ concentration has inhibited almost all the cells. These data suggest that the use of the anticancer Ag-GQDs as nanocarriers to deliver drugs may significantly enhance the efficiency of chemotherapy treatment.

To confirm whether the decrease in the cell viability upon treatment with Ag-GQDs is due to an anticancer property and not a toxic effect, we tested its cytotoxicity in normal
Vero cells after 24 hours of treatment. Figure 7 shows that Ag-GQDs did not affect the cell viability for concentrations up to $150 \mu \mathrm{g} / \mathrm{mL}$ and the half maximal inhibitory concentration $\left(\mathrm{IC}_{50}\right)$ is $375 \mu \mathrm{g} / \mathrm{mL}$. Therefore, our data suggest that the decrease in the viability of the cancer cells is due to an intrinsic anticancer property in Ag-GQDs.

\section{Investigating the mechanism of the cell death responsible for the reduction in the viability of DUI45 and HeLa cells}

In order to elucidate the molecular mechanism responsible for the decrease in the cell viability, we measured the caspase-3/7 activity, which is one of the major effectors in the apoptotic pathways. ${ }^{52}$ DU145 and HeLa cancer cells were treated

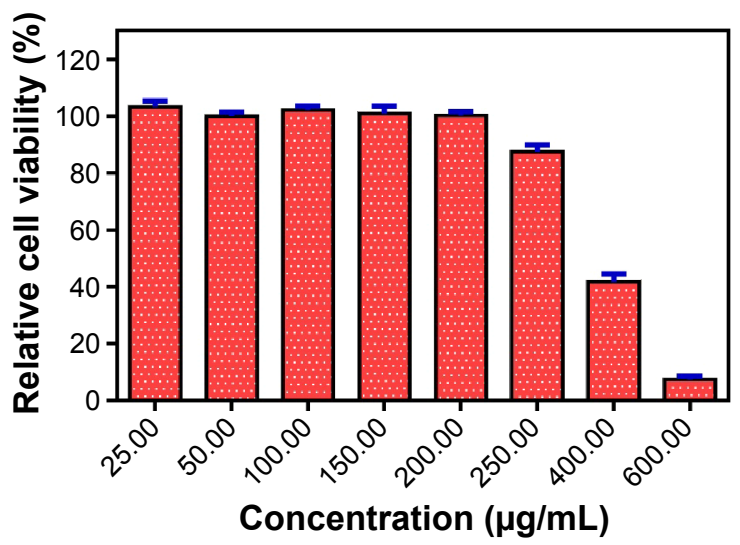

Figure 7 Evaluation of the viability of vero cells treated with Ag-GQDs for 24 hours.

Abbreviation: Ag-GQDs, silver nanoparticles decorated with graphene quantum dots. 

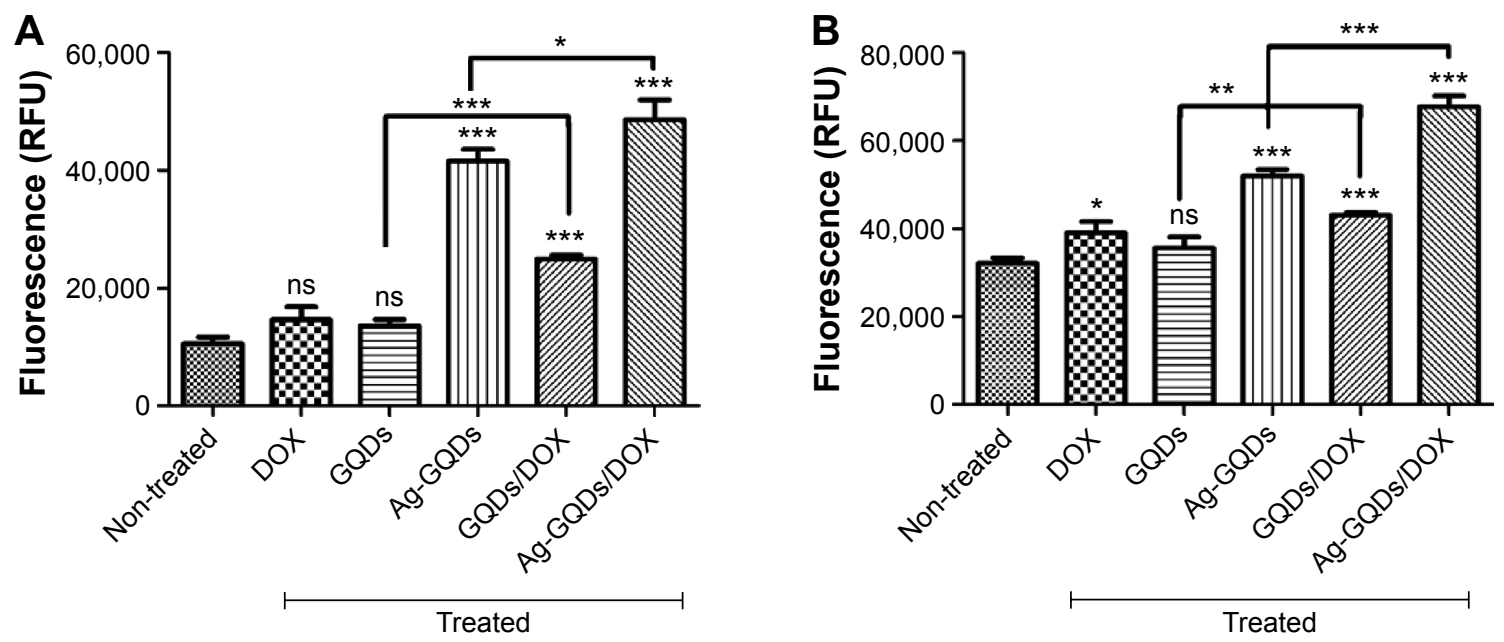

Figure 8 Caspase-3/7 activity was measured as a parameter of apoptotic cell death in: (A) HeLa cells; (B) DUI45 cells.

Notes: The experiment was repeated three times in triplicate. $P$-values are shown as follows: ns, non-significant, $* P<0.05$, $* * P<0.01$, and $* * * P<0.00 \mathrm{I}$ are considered as significant. Abbreviations: Ag-GQDs/DOX, silver nanoparticles decorated with graphene quantum dots conjugated with Doxorubicin; DOX, bare Doxorubicin; Ag-GQDs, silver nanoparticles decorated with graphene quantum dots; GQDs, graphene quantum dots; GQDs/DOX, graphene quantum dots conjugated with Doxorubicin; RFU, relative fluorescence units.

with bare Ag-GQDs, bare GQDs, GQDs/DOX, DOX alone, and Ag-GQDs/DOX for 24 hours. We observed (Figure 8) an increase in the caspase-3/7 activity in both DU145 and HeLa cells suggesting increased levels of apoptosis. It can be noted that treatment with bare DOX and bare GQDs did not induce a significant difference in the activity of caspase-3/7 as compared to the non-treated cells. On the other hand, the bare Ag-GQDs increased the activity of caspase-3/7. Most importantly, the conjugation of Ag-GQDs with a low concentration of DOX $(1 \mu \mathrm{M})$ induced a robust activation of caspase-3/7 in DU145 and HeLa cells. These results confirm the data obtained in the MTS assay, suggesting apoptosis as a major mechanism for the decrease in cell viability.

\section{Cellular uptake and intracellular distribution of Ag-GQDs/DOX in DUI45} In an attempt to evaluate whether Ag-GQDs deliver the drug to the nucleus of the cells or remain in the cytoplasm, we performed fluorescence microscopy imaging of the cells incubated with DOX, Ag-GQDs, and Ag-GQDs/DOX (Figure 9). After 18 hours of treatment of DU145 cells, the cell nuclei were stained, and the cells were studied by excitation and emission fluorescence. We aimed to verify whether the fluorescence of DOX delivered by Ag-GQDs will coincide with the fluorescence of the nuclei's dye or will fluoresce outside the nucleus in the cytoplasm. Figure 9 shows that DOX fluoresces inside the nucleus and confirms that Ag-GQDs diffused into the nucleus. Wang et al postulated that GQDs diffuse to the nucleus of the cells and deliver their cargo of DOX through binding to the DNA. ${ }^{18}$ In the light of the data obtained from HR-TEM and DLS regarding the small size of the Ag-GQDs, it is likely that Ag-GQDs nanoconjugates may penetrate the nucleus by diffusion. Chan et al reported that $60 \mathrm{~nm}$ DNA polylysine complexes, similar to the hydrodynamic size of Ag-GQDs ( $\sim 64 \mathrm{~nm})$, efficiently deliver DNA to the nucleus. ${ }^{53}$ Moreover, we observed that the intensity of the fluorescence of DOX alone is much less than the intensity of the fluorescence of Ag-GQDs/DOX, which indicates that significant amount of DOX is delivered to cells.

\section{Evaluating of the photo-activity of Ag- GQDs and their application in PDT}

It was reported previously that GQDs are excellent PSs for PDT due to the fact that they can generate singlet oxygen via a multistate sensitization process, and they have been used in cancer therapy. ${ }^{16,17}$ We examined if this also applies to Ag-GQDs and whether the decoration of silver nanoparticles with GQDs may offer Ag-NPs the opportunity to act as a PS in PDT. To test the photo-activity of Ag-GQDs in aqueous solution, we used the SOSG, which is a sensitive chemical probe that becomes fluorescent when oxidized by singlet oxygen. The SOSG was mixed with Ag-GQDs and the fluorescence of SOSG was monitored under the photo-irradiation of the mixture with an LED lamp over time (Figure 10A). We used bare GQDs for comparison to determine whether there is an enhancement in the yield of singlet oxygen generated by Ag-GQDs, and PBS mixed with SOSG was used as a control. The singlet oxygen generated by Ag-GQDs in aqueous media (ie, PBS) was observed to be higher than that of bare GQDs, indicating that Ag-GQDs is much better as a PS, as compared to bare GQDs. 

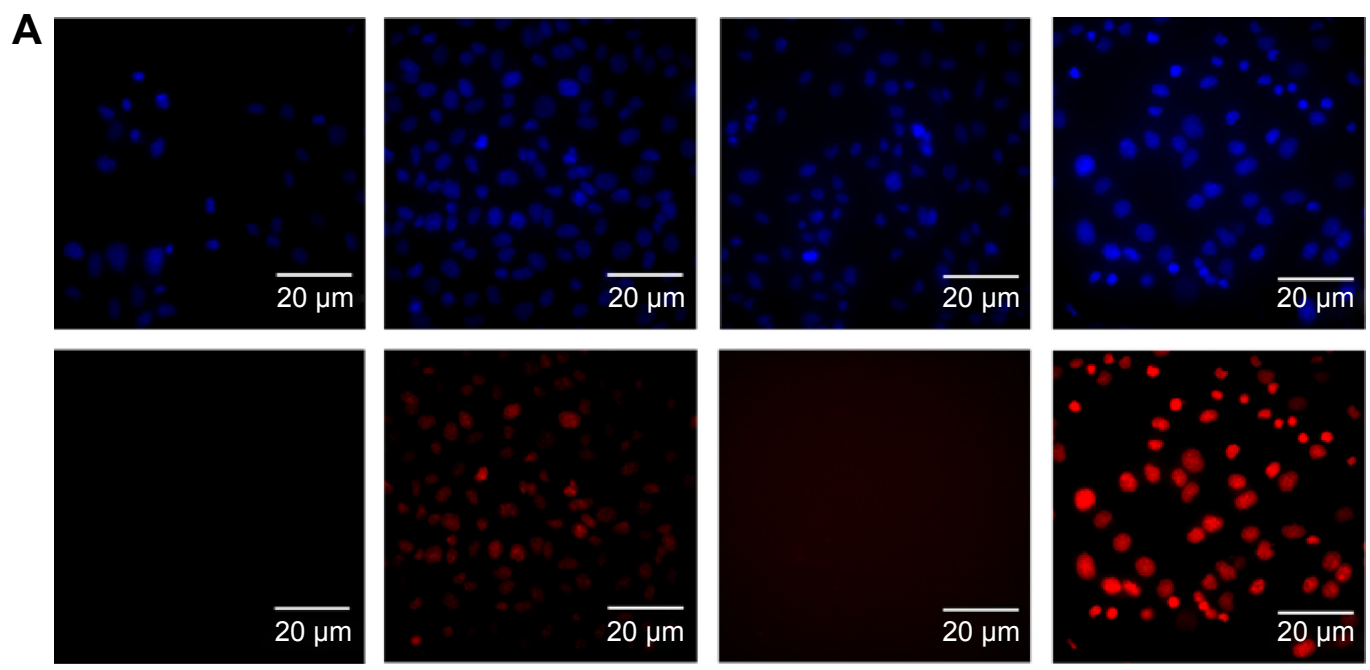

Non-treated

DOX $1 \mu \mathrm{M}$

Ag-GQDs

Ag-GQDs/DOX

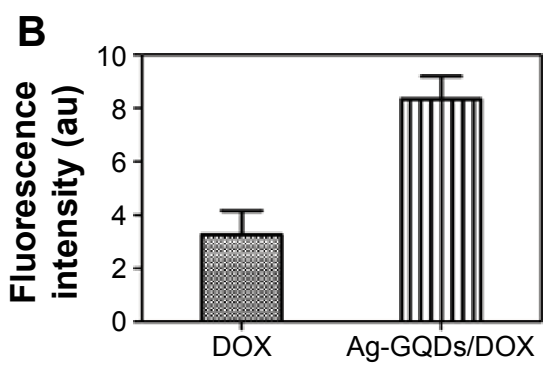

Figure 9 Observation of the cellular uptake and intracellular distribution of DOX delivered by Ag-GQDs nanocomposites.

Notes: (A) Fluorescence images of the DUI 45 cells incubated with Ag-GQDs ( $100 \mu g / \mathrm{mL})$, DOX (I $\mu \mathrm{M})$, and Ag-GQDs/DOX nanoconjugates under the same corresponding DOX and Ag-GQDs concentrations after 18 hours of incubation. (B) Quantitative fluorescence intensities of DOX and Ag-GQDs/DOX nanoconjugates.

Abbreviations: Ag-GQDs/DOX, silver nanoparticles decorated with graphene quantum dots conjugated with doxorubicin; Ag-GQDs, silver nanoparticles decorated with graphene quantum dots; DOX, bare doxorubicin.

To confirm their potential applicability in PDT, we assessed the cell viability test in HeLa and DU145 cells after treatment with Ag-GQDs and GQDs. The cells were treated with the nanoparticles for 18 hours, irradiated with an LED lamp at an excitation wavelength of $425 \pm 10 \mathrm{~nm}$ for 15 minutes, and then incubated for 6 hours again before the evaluation of the cell viability. We observed ( $c f$ Figure 10B and $C$ ) that the maximum reduction achieved in cell viability with the highest concentration of GQDs (ie, $200 \mu \mathrm{g} / \mathrm{mL}$ ) in HeLa and DU145 cells was $\sim 35 \%$ and $15 \%$, respectively. On the other hand, the reduction in the cell viability achieved for HeLa cells treated with Ag-GQDs $(100 \mu \mathrm{g} / \mathrm{mL})$ is $\sim 70 \%$ and $55 \%$ for DU145 cells. The data reveal that the photodynamic treatment with Ag-GQDs reduces the cell proliferation at low doses as compared to other PS (ie, GQDs).

\section{Evaluation of the chemo-photodynamic combined therapy in vitro}

To evaluate whether there is an enhancement in the treatment efficiency when combining both modalities described earlier, we measured the cell viability in both cancer cell lines treated with Ag-GQDs/DOX nanoconjugates (Figure 11). To visualize the difference in treatment efficacy, the cells treated with Ag-GQDs/DOX and irradiated for 15 minutes with a $425 \pm 10 \mathrm{~nm}$ LED lamp with non-treated cells were compared with cells treated by Ag-GQDs under irradiation under the same conditions and cells treated with Ag-GQDs/ DOX at the same concentration used in the combination. It can be observed clearly that the treatment of HeLa cells with the lowest concentration of Ag-GQDs $(75 \mu \mathrm{g} / \mathrm{mL})$ under irradiation alone results in a reduction of $\sim 20 \%$ in the cell viability (Figure 11A). Treatment with Ag-GQDs/DOX alone results in a reduction of $\sim 68 \%$ and the combination of both therapeutic approaches yields a reduction of $\sim 75 \%$ in the cell viability. Thus, there is an enhancement in the treatment efficacy achieved while increasing the cytotoxicity of Ag-GQDs. We also observed an enhancement in the cytotoxic effect of Ag-GQDs/DOX under irradiation with light against DU145 (Figure 11B). Notably, the anticancer effect of Ag-GQDs/DOX in HeLa is stronger than their photo-cytotoxicity at all the concentrations used. However, the photo-cytotoxicity of Ag-GQDs is more dominant in 
Dovepress

Silver-graphene quantum dots in chemo-photodynamic therapy of cancer

A
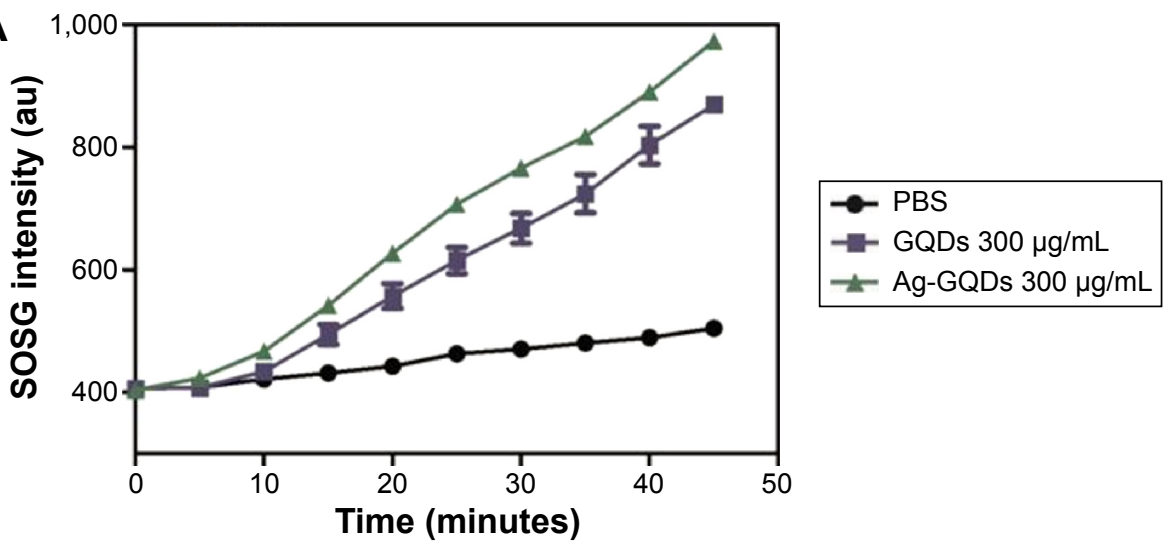

B

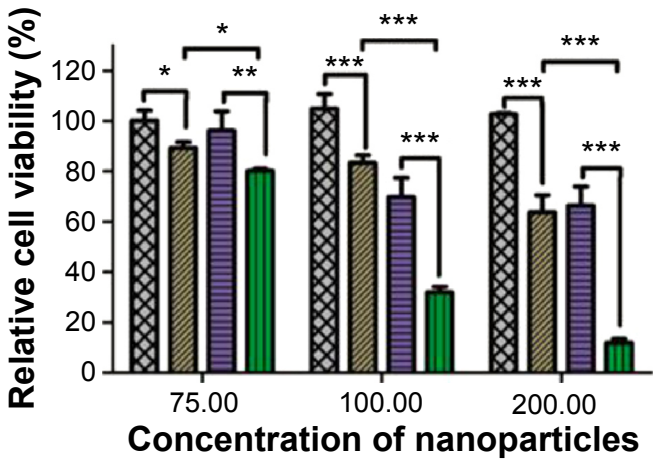
$(\mu \mathrm{g} / \mathrm{mL})$
C

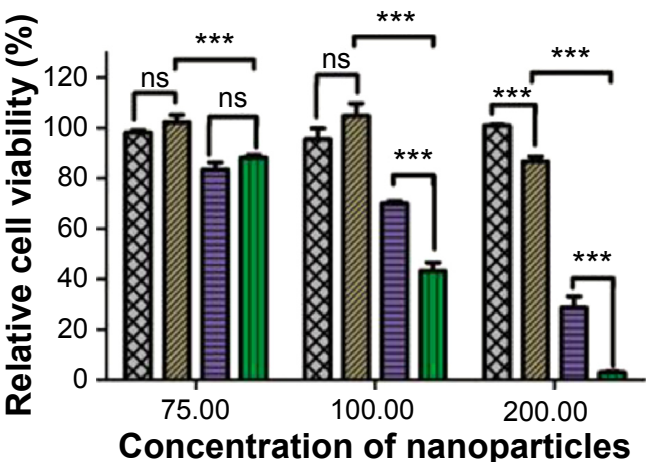

$(\mu \mathrm{g} / \mathrm{mL})$

GODs (dark) 目 Ag-GQDs (dark) GODs (irradiated) 血 Ag-GQDs (irradiated)

Figure 10 Evaluation of the photo-activity of Ag-GQDs nanocomposites.

Notes: (A) Qualitative evaluation of singlet oxygen generated by Ag-GQDs and compared to GQDs and detected by the SOSG. Relative viability of cells treated with AgGQDs and GQDs in dark and under irradiation with a $425 \mathrm{~nm}$ LED lamp for 15 minutes: (B) HeLl and (C) DUI 45 . P-values are shown as follows: ns, nonsignificant, $* P<0.05$, $* * P<0.01$, and $* * * P<0.001$ are considered as significant.

Abbreviations: Ag-GQDs, silver nanoparticles decorated with graphene quantum dots; au, arbitrary unit; GQDs, graphene quantum dots; LED, light emitting diode; SOSG, singlet oxygen sensor green; PBS, phosphate-buffered saline.
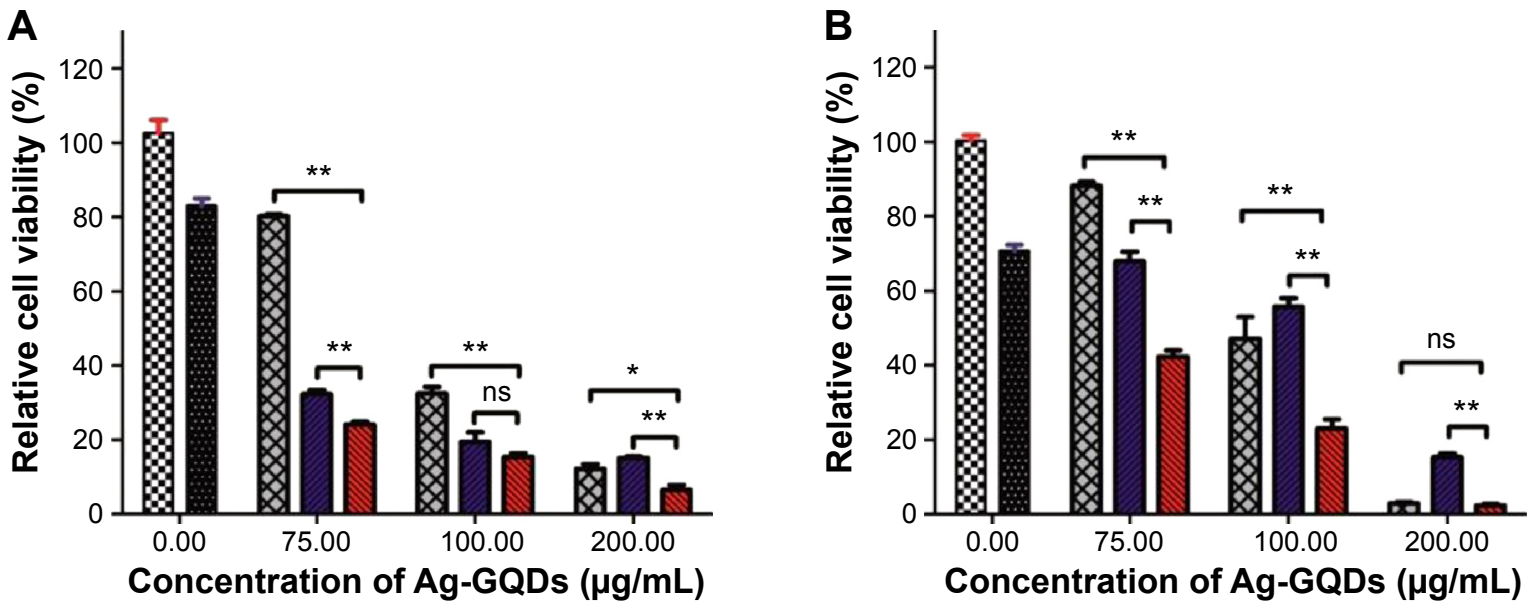

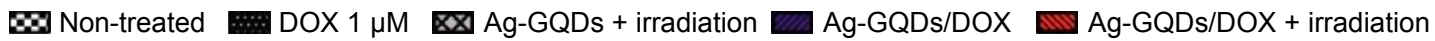

Figure II In vitro relative cell viability of cancer cells treated with Ag-GQDs under irradiation with visible light, Ag-GQDs/DOX in dark or Ag-GQDs/DOX under irradiation with visible light: (A) in HeL cells; (B) in DUI 45 cells.

Notes: $P$-values are shown as follows: ns, nonsignificant, $* P<0.01$, and $* * P<0.001$ are considered as significant.

Abbreviations: Ag-GQDs, silver nanoparticles decorated with grapheme quantum dots; Ag-GQDs/DOX, silver nanoparticles decorated with grapheme quantum dots conjugated with doxorubicin; DOX, bare doxorubicin; GDs, graphene quantum dots; GQDs/DOX, grapheme quantum dots conjugated with doxorubicin.

International Journal of Nanomedicine 2016: II

submit your manuscript

117 
reducing the cell viability of DU145 at almost all the concentrations used, as compared to treatment with Ag-GQDs/ DOX alone. In fact, these data reveal that Ag-GQDs may treat different types of cancer efficiently due to their multimodalities and multiple antitumor activities. It is anticipated that they can be used in the treatment of multi-drug resistant cancer cells through their powerful anti-proliferative properties.

\section{Conclusion}

In summary, we have developed novel, multifunctional, and biocompatible PEGylated Ag-GQDs nanocomposites. The Ag-GQDs effectively delivered DOX, used as a chemotherapy drug model, to HeLa and DU145 cancer cells. They cause an increase in the activity of caspase-3/7 in DU145 and HeLa. Fluorescence imaging results showed that Ag-GQDs deliver their cargo to the nucleus of cancer cells, which suggest that they may deliver genetic material (eg, siRNA and miRNA) in gene therapy. Photo-activation of Ag-GQDs with $425 \mathrm{~nm}$ radiation induces cytotoxicity in cancer cells. The combination of the chemo-photodynamic therapies using Ag-GQDs conjugated with DOX enhanced remarkably the treatment efficacy of HeLa and DU145, as compared to treatment by using each modality alone. The Ag-GQDs thus offer a general platform for incorporating multiple therapeutic modalities (ie, chemotherapy and PDT) for treating different types of cancer and they represent a significant breakthrough in nanomedicine for potential translation to the clinic. Further studies are necessary to elucidate the exact mechanism(s) of Ag-GQDs in releasing their cargo, and to test them in cancer therapy in vivo.

\section{Acknowledgments}

This research was carried out under the auspices of the Institute for Functional Nanomaterials (NSF Cooperative Agreement 1002410), PR NASA EPSCoR (NASA Cooperative Agreement [NNX15AK43A]), PR NASA Space Grant Consortium at University of Puerto RicoBayamon Campus (PRNSGC - UPRB), U54 UPR-MD Anderson Cancer Center Partnership Program (National Cancer Institute through the U54 CA096297/CA096300), and UPR GK-12 Fellowship Program (NSF Grant No. 0841338). We want to acknowledge Mr Oscar Resto from the University of Puerto Rico - Rio Piedras Campus for taking HR-TEM images, Dr Carlos I Gonzalez and Dr Jose A Gonzalez-Feliciano for providing HeLa and DU145 cells, Dr Anthony V Washington for providing access to the fluorescence microscope and Dr Elizabeth Hunsperger from CDC for providing Vero cells.

\section{Author contributions}

All authors contributed toward data analysis, drafting and revising the paper and agree to be accountable for all aspects of the work.

\section{Disclosure}

The authors report no conflicts of interest in this work.

\section{References}

1. Xu C, Yang D, Mei L, Li Q, Zhu H, Wang T. Targeting chemophotothermal therapy of hepatoma by gold nanorods/graphene oxide core/shell nanocomposites. ACS Appl Mater Interfaces. 2013;5(24): $12911-12920$

2. Zhang Z, Wang J, Chen C. Near-infrared light-mediated nanoplatforms for cancer thermo-chemotherapy and optical imaging. Adv Mater. 2013; 25(28):3869-3880

3. Snyder JW, Greco WR, Bellnier DA, Vaughan L, Henderson BW. Photodynamic therapy a means to enhanced drug delivery to tumors. Cancer Res. 2003;63(23):8126-8131.

4. Dolmans DE, Fukumura D, Jain RK. Photodynamic therapy for cancer. Nat Rev Cancer. 2003;3(5):380-387.

5. Chang JE, Yoon IS, Sun PL, Yi E, Jheon S, Shim CK. Anticancer efficacy of photodynamic therapy with hematoporphyrin-modified, doxorubicin-loaded nanoparticles in liver cancer. J Photochem Photobiol B. 2014;140:49-56.

6. Weijer R, Broekgaarden M, Kos M, et al. Enhancing photodynamic therapy of refractory solid cancers: combining second-generation photosensitizers with multi-targeted liposomal delivery. $J$ Photochem Photobiol C: Photochem Rev. 2015;23:103-131.

7. Tolentino Ede S, Centurion BS, Ferreira LcHC, Souza AP, Damante JH, Rubira-Bullen IR. Oral adverse effects of head and neck radiotherapy: literature review and suggestion of a clinical oral care guideline for irradiated patients. J Appl Oral Sci. 2011;19(5):448-454.

8. Demicheli R, Retsky MW, Hrushesky WJM, Baum M, Gukas ID. The effects of surgery on tumor growth: a century of investigations. Ann Oncol. 2008;19:1821-1828.

9. Gao L, Fei J, Zhao J, Li H, Cui Y, Li J. Hypocrellin-loaded gold nanocages with high two-photon efficiency for photothermal/photodynamic cancer therapy in vitro. Acs Nano. 2012;6(9):8030-8040.

10. Tian B, Wang C, Zhang S, Feng L, Liu Z. Photothermally enhanced photodynamic therapy delivered by nano-graphene oxide. Acs Nano. 2011; 5(9):7000-7009.

11. Zhang W, Guo Z, Huang D, Liu Z, Guo X, Zhong H. Synergistic effect of chemo-photothermal therapy using PEGylated graphene oxide. Biomaterials. 2011;32(33):8555-8561.

12. Huang X, Jain PK, El-Sayed IH, El-Sayed MA. Gold nanoparticles: interesting optical properties and recent applications in cancer diagnostics and therapy. Nanomedicine. 2007;2(5):681-693.

13. Khlebtsov N, Bogatyrev V, Dykman L, et al. Analytical and theranostic applications of gold nanoparticles and multifunctional nanocomposites. Theranostics. 2013;3(3):167.

14. Gao X, Cui Y, Levenson RM, Chung LW, Nie S. In vivo cancer targeting and imaging with semiconductor quantum dots. Nat Biotechnol. 2004; 22(8):969-976.

15. Shao L, Gao Y, Yan F. Semiconductor quantum dots for biomedicial applications. Sensors. 2011;11(12):11736-11751.

16. Ge J, Lan M, Zhou B, et al. A graphene quantum dot photodynamic therapy agent with high singlet oxygen generation. Nat Commun. 2014; 5:4596.

17. Markovic ZM, Ristic BZ, Arsikin KM, et al. Graphene quantum dots as autophagy-inducing photodynamic agents. Biomaterials. 2012; 33(29):7084-7092.

18. Wang C, Wu C, Zhou X, et al. Enhancing cell nucleus accumulation and DNA cleavage activity of anti-cancer drug via graphene quantum dots. Sci Rep. 2013;3:2852. 
19. Wang X, Sun X, Lao J, et al. Multifunctional graphene quantum dots for simultaneous targeted cellular imaging and drug delivery. Colloids Surf B Biointerfaces. 2014;122:638-644.

20. Yang K, Feng L, Shi X, Liu Z. Nano-graphene in biomedicine: theranostic applications. Chem Soc Rev. 2013;42(2):530-547.

21. Zhao X, Yang L, Li X, et al. Functionalized graphene oxide nanoparticles for cancer cell-specific delivery of antitumor drug. Bioconjug Chem. 2015;26(1):128-136.

22. Zheng XT, Ananthanarayanan A, Luo KQ, Chen P. Glowing graphene quantum dots and carbon dots: properties, syntheses, and biological applications. Small. 2015;11(14):1620-1636.

23. Janib SM, Moses AS, MacKay JA. Imaging and drug delivery using theranostic nanoparticles. Adv Drug Deliv Rev. 2010;62(11):1052-1063.

24. Yoo D, Lee JH, Shin TH, Cheon J. Theranostic magnetic nanoparticles. Acc Chem Res. 2011;44(10):863-874.

25. Bian X, Song ZL, Qian Y, et al. Fabrication of graphene-isolatedAu-nanocrystal nanostructures for multimodal cell imaging and photothermal-enhanced chemotherapy. Sci Rep. 2014;4:6093.

26. Li JL, Tang B, Yuan B, Sun L, Wang XG. A review of optical imaging and therapy using nanosized graphene and graphene oxide. Biomaterials. 2013;34(37):9519-9534.

27. Liu J, Cui L, Losic D. Graphene and graphene oxide as new nanocarriers for drug delivery applications. Acta Biomater. 2013;9(12): 9243-9257.

28. Qin Y, Zhou ZW, Pan ST, et al. Graphene quantum dots induce apoptosis, autophagy, and inflammatory response via p38 mitogen-activated protein kinase and nuclear factor- $\kappa \mathrm{B}$ mediated signaling pathways in activated THP-1 macrophages. Toxicology. 2015;327:62-76.

29. Sahu A, Choi WI, Lee JH, Tae G. Graphene oxide mediated delivery of methylene blue for combined photodynamic and photothermal therapy. Biomaterials. 2013;34(26):6239-6248.

30. Wang Z, Xia J, Zhou C, et al. Synthesis of strongly green-photoluminescent graphene quantum dots for drug carrier. Colloids Surf B Biointerfaces. 2013;112:192-196.

31. Franco-Molina MA, Mendoza-Gamboa E, Sierra-Rivera CA, et al. Antitumor activity of colloidal silver on MCF-7 human breast cancer cells. J Exp Clin Cancer Res. 2010;29(1):148.

32. Mazurak VC, Burrell RE, Tredget EE, Clandinin MT, Field CJ. The effect of treating infected skin grafts with Acticoat ${ }^{\mathrm{TM}}$ on immune cells. Burns. 2007;33(1):52-58.

33. Sanpui P, Chattopadhyay A, Ghosh SS. Induction of apoptosis in cancer cells at low silver nanoparticle concentrations using chitosan nanocarrier. ACS Appl Mater Interfaces. 2011;3(2):218-228.

34. AshaRani PV, Low Kah Mun G, Hande MP, Valiyaveettil S. Cytotoxicity and genotoxicity of silver nanoparticles in human cells. Acs Nano. 2008; 3(2):279-290.

35. Krolow MZ, Hartwig CA, Link GC, et al, editors. Synthesis and Characterisation of Carbon Nanocomposites. In: NanoCarbon 2011. Berlin Heidelberg: Springer; 2013:33-47.

36. Seo Y, Hwang J, Kim J, Jeong Y, Hwang MP, Choi J. Antibacterial activity and cytotoxicity of multi-walled carbon nanotubes decorated with silver nanoparticles. Int J Nanomedicine. 2014;9:4621-4629.
37. Sachdev A, Matai I, Gopinath P. Dual-functional carbon dots-silver@ zinc oxide nanocomposite: in vitro evaluation of cellular uptake and induction of apoptosis. J Mater Chem B. 2015;3(7):1217-1229.

38. Wang X, Wang C, Cheng L, Lee ST, Liu Z. Noble metal coated singlewalled carbon nanotubes for applications in surface enhanced raman scattering imaging and photothermal therapy. J Am Chem Soc. 2012; 134(17):7414-7422.

39. Jokerst JV, Lobovkina T, Zare RN, Gambhir SS. Nanoparticle PEGylation for imaging and therapy. Nanomedicine. 2011;6(4):715-728.

40. Li X, Wang L, Fan Y, Feng Q, Cui FZ. Biocompatibility and toxicity of nanoparticles and nanotubes. J Nanomater. 2012;2012:6.

41. Habiba K, Makarov VI, Avalos J, Guinel MJ, Weiner BR, Morell G. Luminescent graphene quantum dots fabricated by pulsed laser synthesis. Carbon. 2013;64:341-350.

42. Habiba K, Makarov VI, Weiner BR, Morell G. Fabrication of nanomaterials by pulsed laser synthesis. In: Ahmed W, Ali N, editors. Manufacturing Nanostructures. Manchester: One Central Press (OCN); 2014: 263-292.

43. Sanyakamdhorn S, Agudelo D, Tajmir-Riahi HA. Encapsulation of antitumor drug doxorubicin and its analogue by chitosan nanoparticles. Biomacromolecules. 2013;14(2):557-563.

44. Theivasanthi T, Alagar M. Electrolytic synthesis and characterizations of silver nanopowder. Nano Biomed Eng. 2012;2012(4):58-65.

45. Liu Q, Guo B, Rao Z, Zhang B, Gong JR. Strong two-photon-induced fluorescence from photostable, biocompatible nitrogen-doped graphene quantum dots for cellular and deep-tissue imaging. Nano Lett. 2013; 13(6):2436-2441.

46. El Moussaoui A, Chauvet A, Masse J. Etude des interactions a l'etat solide du nordazepam III-polyoxyethylene glycol 6000 et nordazepam III-acide succinique. J Therm Anal Calorim. 1993;39(3):373-392.

47. Liu R, Wu D, Feng X, Müllen K. Bottom-up fabrication of photoluminescent graphene quantum dots with uniform morphology. J Am Chem Soc. 2011;133(39):15221-15223.

48. Serra A, Filippo E, Re M, et al. Non-functionalized silver nanoparticles for a localized surface plasmon resonance-based glucose sensor. Nanotechnology. 2009;20(16):165501.

49. Dreaden EC, Austin LA, Mackey MA, El-Sayed MA. Size matters: gold nanoparticles in targeted cancer drug delivery. Ther Deliv. 2012;3(4): $457-478$.

50. Li J, Yu X, Wang Y, et al. A reduction and $\mathrm{pH}$ dual-sensitive polymeric vector for long-circulating and tumor-targeted siRNA delivery. Adv Mater. 2014;26(48):8217-8224.

51. Honary S, Zahir F. Effect of zeta potential on the properties of nanodrug delivery systems-a review (Part 2). Trop J Pharm Res. 2013;12(2) 265-273.

52. Walsh JG, Cullen SP, Sheridan C, Lüthi AU, Gerner C, Martin SJ. Executioner caspase-3 and caspase-7 are functionally distinct proteases. Proc Natl Acad Sci U S A. 2008;105(35):12815-12819.

53. Chan CK, Senden T, Jans DA. Supramolecular structure and nuclear targeting efficiency determine the enhancement of transfection by modified polylysines. Gene Ther. 2000;7(19):1690-1697.
International Journal of Nanomedicine

\section{Publish your work in this journal}

The International Journal of Nanomedicine is an international, peerreviewed journal focusing on the application of nanotechnology in diagnostics, therapeutics, and drug delivery systems throughout the biomedical field. This journal is indexed on PubMed Central, MedLine, CAS, SciSearch $®$, Current Contents $\AA /$ Clinical Medicine,

\section{Dovepress}

Journal Citation Reports/Science Edition, EMBase, Scopus and the Elsevier Bibliographic databases. The manuscript management system is completely online and includes a very quick and fair peer-review system, which is all easy to use. Visit http://www.dovepress.com/ testimonials.php to read real quotes from published authors. 